\title{
Semi-Blind Time-Domain Equalization for MIMO-OFDM Systems
}

\author{
Shaodan Ma and Tung-Sang Ng, Fellow, IEEE
}

\begin{abstract}
In this paper, a semi-blind time-domain equalization technique is proposed for general multiple-input-multiple-output (MIMO) orthogonal frequency-division multiplexing (OFDM) systems. The received OFDM symbols are shifted by more than or equal to the cyclic prefix (CP) length, and a blind equalizer is designed to completely suppress both intercarrier interference (ICI) and intersymbol interference (ISI) using second-order statistics of the shifted received OFDM symbols. Only a one-tap equalizer is needed to detect the time-domain signals from the blind equalizer output, and one pilot OFDM symbol is utilized to estimate the required channel state information for the design of the one-tap equalizer. The technique is applicable irrespective of whether the CP length is longer than, equal to, or shorter than the channel length. Computer simulations show that the proposed technique outperforms the existing techniques, and it is robust against the number of shifts in excess of the CP length.
\end{abstract}

Index Terms-Equalization, multiple-input-multiple-output (MIMO), orthogonal frequency-division multiplexing (OFDM), second-order statistics (SOS).

\section{INTRODUCTION}

$\mathbf{T}$ O ACHIEVE a high system capacity for multimedia applications in wireless communications, various methods have been proposed in recent years. Among them, the multipleinput-multiple-output (MIMO) system using multiple antennas at both the transmitter and the receiver has attracted a lot of research interest due to its potential to increase the system capacity without extra bandwidth [1]-[4]. Previous work has shown that the system capacity could be linearly increased with the number of antennas when the system is operating over flat fading channels [1], [2]. In real situations, multipath propagation usually occurs and causes the MIMO channels to be frequency selective. To combat the effect of frequencyselective fading, MIMO is generally combined with orthogonal frequency-division multiplexing (OFDM) technique, which transforms the frequency-selective fading channels into parallel flat fading subchannels, as long as the cyclic prefix (CP) inserted at the beginning of each OFDM symbol is longer than or equal to the channel length [5]-[17]. In this case, the signals on each subcarrier can be easily detected by a one-tap frequency-

Manuscript received March 15, 2006; revised March 31, 2007 and September 22, 2007; accepted September 25, 2007. This work was supported by the Hong Kong Research Grants Council under Grant 7164/04E. The review of this paper was coordinated by Prof. M. Juntti.

The authors are with the Department of Electrical and Electronic Engineering, The University of Hong Kong, Hong Kong (e-mail: sdma@eee.hku.hk; tsng@eee.hku.hk).

Digital Object Identifier 10.1109/TVT.2007.912331 domain equalizer. In cases where a short $\mathrm{CP}$ is inserted for increasing bandwidth efficiency, or because of some unforeseen channel behavior, the $\mathrm{CP}$ length is shorter than the channel length. In this case, the effect of frequency-selective fading cannot be completely eliminated, and intercarrier interference (ICI) and intersymbol interference (ISI) will be introduced. Equalization techniques that could flexibly detect the signals in both cases are thus important in MIMO-OFDM systems.

The issue of equalization for MIMO-OFDM systems has been widely discussed in [18]-[24]. In general, there are three categories of equalization techniques. The first one is the frequency-domain technique [18], [19], which applies the conventional equalization algorithm for single-carrier MIMO systems to each subcarrier after modeling the smoothed persubcarrier received signal similarly to the smoothed received signal of a single-carrier MIMO system [19]. Since a different equalizer is needed for each subcarrier, the design complexity is rather high, and the memory required to store the equalizer coefficients is large. The second technique is the time-frequency domain equalization with channel shortening [20]-[23]. A time-domain equalizer is inserted to reduce the MIMO channels to the ones with the channel length shorter than or equal to the CP length, and then, a one-tap frequency-domain equalizer is applied to each subcarrier. When the MIMO channels are shortened by the time-domain equalizer, residual ICI and ISI are introduced. They cannot be eliminated by the subsequent frequency-domain equalizer and, thus, limit the performance. Moreover, it has been shown that time-frequency domain equalization techniques with channel shortening are sensitive to parameters, including the channel-shortening equalizer length and the delay [22], [25]. The third technique is the time-domain statistics-based technique previously proposed by the authors in [24]. The method is based on some structural properties of the received OFDM symbols, which are shifted within the $C P$ length. A time-domain equalizer, which is designed using the second-order statistics (SOS) of the shifted received OFDM symbols, is applied to partially cancel the ICI and ISI. The equalizer output contains two sampled signals from each transmit antenna, and the time-domain signals are then detected with the aid of two pilot OFDM symbols.

In this paper, the case when the received OFDM symbols are shifted by more than or equal to the CP length is considered, assuming that prefect time and frequency synchronization has been achieved. It turns out that the SOS matrices of the shifted received OFDM symbols have different and useful structures. With these structures, a blind equalizer can be designed to completely suppress the ICI and ISI using the SOS of the shifted received OFDM symbols. Unlike [24], the equalizer 


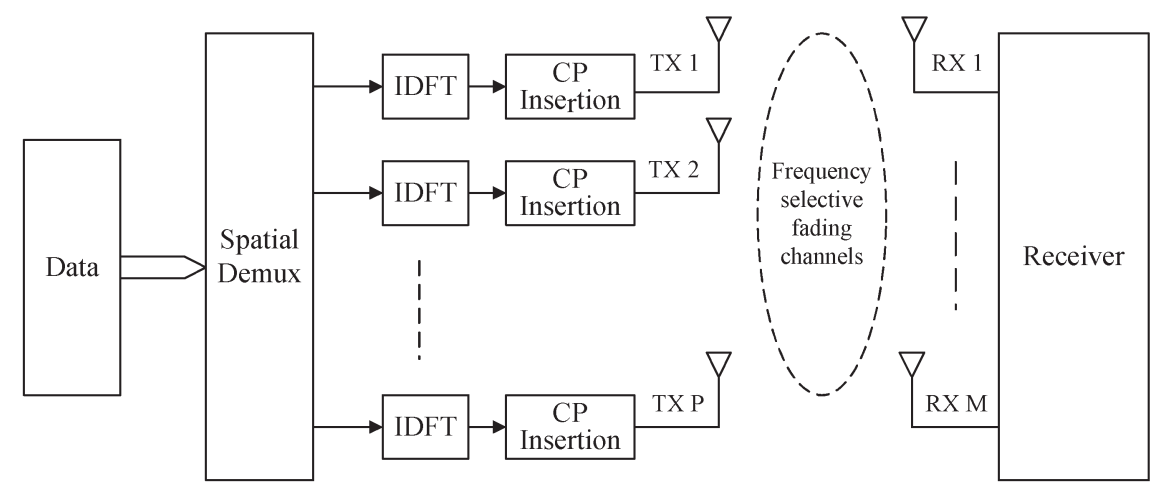

Fig. 1. MIMO-OFDM system.

output contains only one sampled signal from each transmit antenna. Consequently, only a one-tap equalizer is needed to detect the time-domain signals, and one pilot OFDM symbol is utilized to estimate the required channel state information for the design of the one-tap equalizer, given that the number of transmit antennas is less than the number of subcarriers in one OFDM symbol.

Compared with the existing frequency-domain techniques [18], [19], the proposed technique has a lower design complexity and a smaller memory requirement. Compared with the existing time-frequency domain techniques with channel shortening [20]-[23], there is no residual ICI and ISI. In addition, only one parameter (the number of shifts in excess of the CP length, $K$ ) can be varied, and simulation results show that the proposed technique is robust against variation of $K$, whereas the time-frequency domain techniques are sensitive to a number of parameters, such as the channel-shortening equalizer length and the delay.

The rest of this paper is organized as follows: Section II introduces the shifted signal model of the MIMO-OFDM system. Section III presents some properties of the signals and channel matrix to be used for equalization, and Section IV proposes the semi-blind time-domain equalization technique. In Section V, the performance of the proposed technique is investigated in both cases where the $\mathrm{CP}$ length is longer than/equal to or shorter than the channel length by computer simulations. It is shown that the proposed technique performs well in both cases. Finally, this paper is concluded in Section VI.

Notation: Superscripts $(\bullet)^{T},(\bullet)^{*}$, and $(\bullet)^{\#}$ represent transpose, conjugate transpose, and pseudoinverse operations, respectively; $\otimes, E\{\bullet\}$, and $\mathbf{I}_{a}$ denote the Kronecker product of matrices, the statistical mean, and the $a \times a$ identity matrix, respectively.

\section{SYSTEM MODEL}

Consider a MIMO-OFDM system with $P$ transmit antennas and $M$ receive antennas, as illustrated in Fig. 1. To achieve a high throughput, spatial multiplexing is applied here, and independent data streams are transmitted through different antennas. Before transmission, each data stream is modulated by $N$-point inverse discrete Fourier transform (IDFT), and a CP with length of $L_{\mathrm{CP}}$ is inserted at the beginning of each OFDM symbol. Denote the $i$ th block signal from the $p$ th transmit antenna before OFDM modulation as

$$
\begin{aligned}
& \boldsymbol{\beta}_{i, p}=\left[\begin{array}{llll}
\beta_{i, p}[0] & \beta_{i, p}[1] & \cdots & \beta_{i, p}[N-1]
\end{array}\right]^{T} \\
& p \\
& p \in\{1,2, \ldots, P\}
\end{aligned}
$$

which is the so-called frequency-domain signal vector. Here, the frequency-domain signal $\beta_{i, p}[n], n \in\{0,1, \ldots, N-1\}$, is assumed to be white with zero mean and unit variance. Performing $N$-point IDFT, the so-called time-domain signal vector is generated as

$$
\begin{aligned}
& \mathbf{b}_{i, p}=\left[b_{i, p}[0] \quad b_{i, p}[1] \quad \cdots \quad b_{i, p}[N-1]\right]^{T}=\mathbf{F}_{N} \boldsymbol{\beta}_{i, p} \\
& p \in\{1,2, \ldots, P\}
\end{aligned}
$$

where $\mathbf{F}_{N}$ is the $N \times N$ IDFT matrix with the $(n+1, k+1)$ th entry being $e^{j 2 \pi n k / N} / \sqrt{N}, n, k \in\{0,1, \ldots, N-1\}$. After the CP insertion, the transmitted $i$ th OFDM symbol from the $p$ th transmit antenna is

$$
\mathbf{s}_{i, p}=\left[s_{i, p}[0] \quad s_{i, p}[1] \quad \cdots \quad s_{i, p}\left[N^{\prime}-1\right]\right]^{T}
$$

where $N^{\prime}=N+L_{\mathrm{CP}}$, and

$$
s_{i, p}[n]= \begin{cases}b_{i, p}\left[n-L_{\mathrm{CP}}+N\right], & 0 \leq n \leq L_{\mathrm{CP}}-1 \\ b_{i, p}\left[n-L_{\mathrm{CP}}\right], & L_{\mathrm{CP}} \leq n \leq N^{\prime}-1 .\end{cases}
$$

Each OFDM symbol is then simultaneously transmitted over quasi-static frequency-selective fading MIMO channels. Generally, the frequency-selective fading channel is modeled as an $L$-tap FIR filter, so that the $M \times 1$ sampled received signal vector in the $i$ th received OFDM symbol is written as

$$
\mathbf{y}_{i}[n]=\sum_{p=1}^{P} \sum_{l=0}^{L} \mathbf{h}_{p}(l) s_{i, p}[n-l]+\mathbf{w}_{i}[n], \quad n=0,1, \ldots, N^{\prime}-1
$$

where $\mathbf{h}_{p}(l)$ is an $M \times 1$ channel response vector; $\mathbf{w}_{i}[n]$ represents the $M \times 1$ additive noise vector; and $s_{i, p}[n]=$ $s_{(i-1), p}\left[N^{\prime}+n\right]$ when $n<0$, and $s_{i, p}[n]=s_{(i+1), p}\left[n-N^{\prime}\right]$ when $N^{\prime} \leq n$. Without loss of generality, the channel length is assumed to be far less than the number of subcarriers in one OFDM symbol, i.e., $L \ll N$. The noise is assumed to be independent of the transmitted signals $s_{i, p}[n]$ and is independently 
identically distributed complex Gaussian with zero mean and variance $\sigma^{2}$.

Similarly to [24], the CP is not discarded, and $N^{\prime}$ sampled received signal vectors are collected at the receiver as

$$
\begin{array}{r}
\mathbf{y}_{i}^{(k)}=\left[\begin{array}{lll}
\mathbf{y}_{i}[-k]^{T} & \mathbf{y}_{i}[-k+1
\end{array}\right]^{T} \quad \cdots \quad \mathbf{y}_{i}\left[N^{\prime}-1-k\right]^{T} \\
{ }^{T} \\
k=0, \pm 1, \pm 2, \ldots
\end{array}
$$

where $\mathbf{y}_{i}[n]$ corresponds to the signal at the $(i-1)$ th received OFDM symbol and is equal to $\mathbf{y}_{i-1}\left[n+N^{\prime}\right]$ when $n<0$, whereas it corresponds to the signal at the $(i+1)$ th received OFDM symbol and is equal to $\mathbf{y}_{i+1}\left[n-N^{\prime}\right]$ when $N^{\prime} \leq n$. In fact, the received signal vector $\mathbf{y}_{i}^{(k)}$ corresponds to the $i$ th received OFDM symbol shifted by $k$ samples and can be expressed as

$$
\mathbf{y}_{i}^{(k)}=\mathbf{H} \mathbf{x}_{i}^{(k)}+\mathbf{w}_{i}^{(k)}, \quad k=0, \pm 1, \pm 2, \ldots
$$

where $\mathbf{H}, \mathbf{x}_{i}^{(k)}$, and $\mathbf{w}_{i}^{(k)}$ are given in (8)-(12), shown at the bottom of the page. Here, the channel matrix $\mathbf{H}$ is assumed to be of full column rank after removing all-zero columns. This assumption is a sufficient condition to detect the time-domain signals based on SOS of the received signal vector $\mathbf{y}_{i}^{(k)}$ [7], [27] and is generally consistent with real situations [7].

\section{Properties of the Transmitted Signals AND CHANNEL MATRIX}

The following properties of the transmitted signals and channel matrix in the MIMO-OFDM system under consideration will be used to derive the proposed semi-blind equalization method.

Property 1: The transmitted signal $s_{i, p}[n]$ satisfies

$$
\begin{gathered}
E\left\{s_{i_{1}, p_{1}}\left[n_{1}\right] s_{i_{2}, p_{2}}\left[n_{2}\right]^{*}\right\} \\
= \begin{cases}1, & i_{1}=i_{2} \text { and } p_{1}=p_{2} \text { and }\left(n_{1}=n_{2} \text { or }\left|n_{1}-n_{2}\right|=N\right) \\
0, & \text { otherwise }\end{cases} \\
n_{1}, n_{2} \in\left\{0,1, \ldots, N^{\prime}-1\right\} .
\end{gathered}
$$

Proof: Since independent data streams are transmitted through different antennas and the frequency-domain signal $\beta_{i, p}[n], n \in\{0,1, \ldots, N-1\}$ is assumed to be white with zero mean and unit variance, it follows that the time-domain signals $b_{i, p}[n]$ (2) are uncorrelated as the IDFT matrix $\mathbf{F}_{N}$ is unitary, and the uncorrelated property of the signal vector is maintained by unitary transformation. It follows that $b_{i, p}[n]=$ $\mathbf{F}_{N}(n+1) \boldsymbol{\beta}_{i, p}$ and

$$
\begin{gathered}
E\left\{b_{i_{1}, p_{1}}\left[n_{1}\right] b_{i_{2}, p_{2}}\left[n_{2}\right]^{*}\right\} \\
=E\left\{\mathbf{F}_{N}\left(n_{1}+1\right) \boldsymbol{\beta}_{i_{1}, p_{1}} \boldsymbol{\beta}_{i_{2}, p_{2}}^{*} \mathbf{F}_{N}\left(n_{2}+1\right)^{*}\right\} \\
= \begin{cases}1, & i_{1}=i_{2} \text { and } p_{1}=p_{2} \text { and } n_{1}=n_{2} \\
0, & \text { otherwise }\end{cases} \\
n_{1}, n_{2} \in\{0,1, \ldots, N-1\}
\end{gathered}
$$

where $\mathbf{F}_{N}(a)$ denotes the $a$ th row of the IDFT matrix $\mathbf{F}_{N}$. From the structure of the OFDM symbol with $\mathrm{CP}$ in (4) and the cyclic property of $\mathrm{CP}$ (which means that the $\mathrm{CP}$ is the repeated signals of the last $L_{\mathrm{CP}}$ time-domain signals $b_{i, p}[N-$ $\left.\left.L_{\mathrm{CP}}\right], \ldots, b_{i, p}[N-1]\right)$, the property follows.

Property 2: The channel matrix $\mathbf{H}$ satisfies

$$
\mathbf{H}^{*}\left(\mathbf{H H}^{*}\right)^{\#} \mathbf{H}=\mathbf{A}_{\left(N^{\prime}+L\right) P}
$$

where $\mathbf{A}_{\left(N^{\prime}+L\right) P}$ is an $\left(N^{\prime}+L\right) P \times\left(N^{\prime}+L\right) P$ identity matrix with all-zero rows corresponding to all-zero columns of $\mathbf{H}$.

Proof: Under the full column rank assumption of the channel matrix $\mathbf{H}$, the result directly follows from [26].

\section{Semi-Blind Time-Domain Equalization}

It is obvious from (7) that the received signal vector $\mathbf{y}_{i}^{(k)}$ is a time-domain signal vector with multiantenna interference (MAI), ICI, and ISI. In this section, a time-domain equalization technique is proposed, as illustrated in Fig. 2. It is a semi-blind technique since both blind information (the SOS of the received signals), and one pilot symbol will be utilized to design the equalizers.

$$
\begin{aligned}
& \mathbf{H}=\left[\begin{array}{llll}
\mathbf{H}_{1} & \mathbf{H}_{2} & \cdots & \mathbf{H}_{P}
\end{array}\right] \\
& \mathbf{H}_{p}=\left[\begin{array}{ccccccc}
\mathbf{h}_{p}(L) & \mathbf{h}_{p}(L-1) & \cdots & \mathbf{h}_{p}(0) & \mathbf{0} & \cdots & \mathbf{0} \\
\mathbf{0} & \mathbf{h}_{p}(L) & \mathbf{h}_{p}(L-1) & \cdots & \mathbf{h}_{p}(0) & \ddots & \mathbf{0} \\
\vdots & \ddots & \ddots & \ddots & \ddots & \ddots & \vdots \\
\mathbf{0} & \cdots & \mathbf{0} & \mathbf{h}_{p}(L) & \mathbf{h}_{p}(L-1) & \cdots & \mathbf{h}_{p}(0)
\end{array}\right], \quad p \in\{1,2, \cdots, P\} \\
& \mathbf{x}_{i}^{(k)}=\left[\begin{array}{llll}
\left(\mathbf{x}_{i, 1}^{(k)}\right)^{T} & \left(\mathbf{x}_{i, 2}^{(k)}\right)^{T} & \cdots & \left(\mathbf{x}_{i, P}^{(k)}\right)^{T}
\end{array}\right]^{T} \\
& \mathbf{x}_{i, p}^{(k)}=\left[\begin{array}{lllll}
s_{i, p}[-L-k] & \cdots & s_{i, p}[0] & \cdots & s_{i, p}\left[N^{\prime}-1-k\right]
\end{array}\right]^{T}, \quad p \in\{1,2, \ldots, P\} \\
& \left.\mathbf{w}_{i}^{(k)}=\left[\begin{array}{lll}
\mathbf{w}_{i}[-k & ]^{T} & \mathbf{w}_{i}[-k+1
\end{array}\right]^{T} \quad \cdots \quad \mathbf{w}_{i}\left[N^{\prime}-1-k\right]^{T}\right]^{T}
\end{aligned}
$$




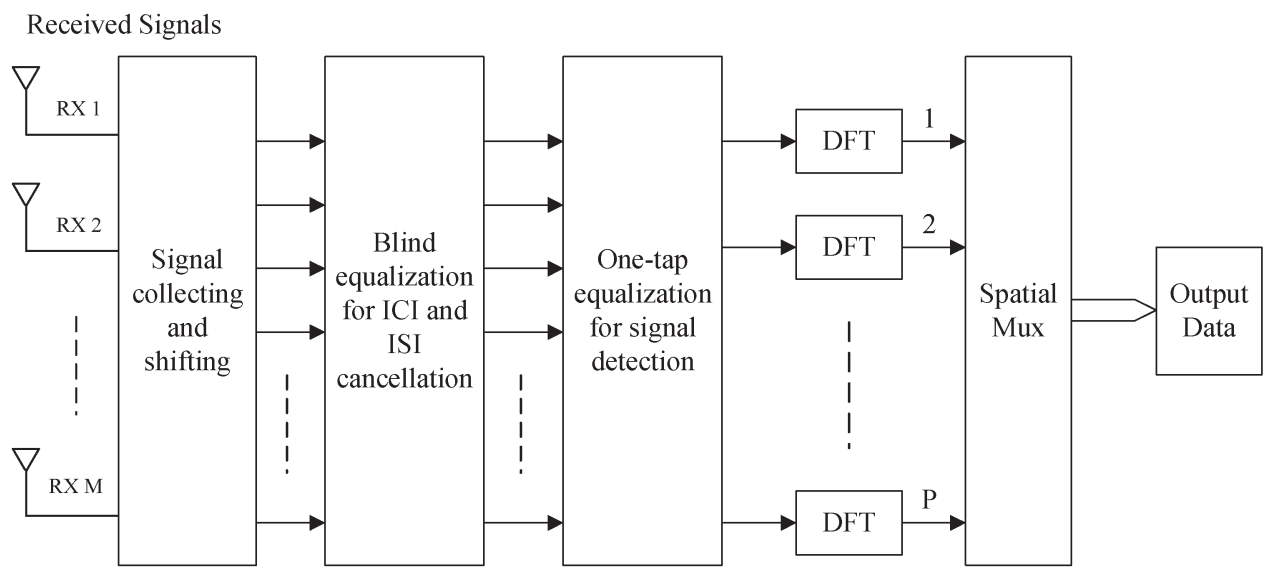

Fig. 2. Receiver structure.

\section{A. Blind Equalization}

Now, consider the autocorrelation matrices of the received signal vector $\mathbf{y}_{i}^{(k)}$ in (7), and define

$$
\begin{aligned}
\mathbf{R}_{y}(0)= & E\left\{\mathbf{y}_{i}^{\left(L_{\mathrm{CP}}\right)} \mathbf{y}_{i}^{\left(L_{\mathrm{CP}}\right)^{*}}\right\} \\
= & \mathbf{H} E\left\{\mathbf{x}_{i}^{\left(L_{\mathrm{CP}}\right)} \mathbf{x}_{i}^{\left(L_{\mathrm{CP}}\right)^{*}}\right\} \mathbf{H}^{*}+E\left\{\mathbf{w}_{i}^{\left(L_{\mathrm{CP}}\right)} \mathbf{w}_{i}^{\left(L_{\mathrm{CP}}\right)^{*}}\right\} \\
\mathbf{R}_{y}(K)= & E\left\{\mathbf{y}_{i}^{\left(L_{\mathrm{CP}}+K\right)} \mathbf{y}_{i}^{\left(L_{\mathrm{CP}}\right)^{*}}\right\} \\
= & \mathbf{H} E\left\{\mathbf{x}_{i}^{\left(L_{\mathrm{CP}}+K\right)} \mathbf{x}_{i}^{\left(L_{\mathrm{CP}}\right)^{*}}\right\} \mathbf{H}^{*} \\
& +E\left\{\mathbf{w}_{i}^{\left(L_{\mathrm{CP}}+K\right)} \mathbf{w}_{i}^{\left(L_{\mathrm{CP}}\right)^{*}}\right\}, \quad 0 \leq K \leq L_{\mathrm{CP}} \\
\mathbf{R}_{y}(-K)= & E\left\{\mathbf{y}_{i}^{\left(L_{\mathrm{CP}}\right)} \mathbf{y}_{i}^{\left(L_{\mathrm{CP}}+K\right)^{*}}\right\} \\
= & \mathbf{H} E\left\{\mathbf{x}_{i}^{\left(L_{\mathrm{CP}}\right)} \mathbf{x}_{i}^{\left(L_{\mathrm{CP}}+K\right)^{*}}\right\} \mathbf{H}^{*} \\
& +E\left\{\mathbf{w}_{i}^{\left(L_{\mathrm{CP})}\right)} \mathbf{w}_{i}^{\left(L_{\mathrm{CP}}+K\right)^{*}}\right\}, \quad 0 \leq K \leq L_{\mathrm{CP}} .
\end{aligned}
$$

Unlike the time-domain statistics-based technique [24], which considers the autocorrelation matrices of $\mathbf{y}_{i}^{(0)}$ and $\mathbf{y}_{i}^{(1)}$, the autocorrelation matrices of $\mathbf{y}_{i}^{\left(L_{\mathrm{CP}}\right)}$ and $\mathbf{y}_{i}^{\left(L_{\mathrm{CP}}+K\right)}$ are utilized here. It is apparent that $\mathbf{y}_{i}^{\left(L_{\mathrm{CP}}\right)}$ and $\mathbf{y}_{i}^{\left(L_{\mathrm{CP}}+K\right)}$, respectively, correspond to the $i$ th received OFDM symbol shifted by $L_{\mathrm{CP}}$ and $L_{\mathrm{CP}}+K$ samples. It follows that $\mathbf{R}_{y}(0), \mathbf{R}_{y}(K)$, and $\mathbf{R}_{y}(-K)$ are the autocorrelation matrices of the received OFDM symbols shifted by more than or equal to the CP length.
Here, the parameter $K$ represents the number of shifts in excess of the CP length.

Based on Property 1, (10), and (11), the signal vectors $\mathbf{x}_{i}^{\left(L_{\mathrm{CP}}\right)}$ and $\mathbf{x}_{i}^{\left(L_{\mathrm{CP}}+K\right)}$ in (16)-(18) satisfy

$$
\begin{aligned}
E\left\{\mathbf{x}_{i}^{\left(L_{\mathrm{CP}}\right)} \mathbf{x}_{i}^{\left(L_{\mathrm{CP}}\right)^{*}}\right\} & =\mathbf{I}_{\left(N^{\prime}+L\right) P} \\
E\left\{\mathbf{x}_{i}^{\left(L_{\mathrm{CP}}+K\right)} \mathbf{x}_{i}^{\left(L_{\mathrm{CP}}\right)^{*}}\right\} & =\mathbf{I}_{P} \otimes \mathbf{J}^{K} \\
E\left\{\mathbf{x}_{i}^{\left(L_{\mathrm{CP}}\right)} \mathbf{x}_{i}^{\left(L_{\mathrm{CP}}+K\right)^{*}}\right\} & =\mathbf{I}_{P} \otimes \mathbf{J}^{-K}
\end{aligned}
$$

where $\mathbf{J}^{K}$ denotes an $\left(N^{\prime}+L\right) \times\left(N^{\prime}+L\right)$ matrix with zero entries except along the lower $K$ th subdiagonal, in which the entries are one; $\mathbf{J}^{-K}$ is equal to $\left(\mathbf{J}^{K}\right)^{*}$. According to the definition of the matrix $\mathbf{J}^{K}$, it satisfies (22) and (23), shown at the bottom of the page [28], where $\mathbf{0}_{a \times b}$ is an $a \times b$ zero matrix. The matrix $\mathbf{J}^{-K} \mathbf{J}^{K}-\mathbf{J}^{-(K+1)} \mathbf{J}^{(K+1)}$ in (23) is zero, except for the $\left(N^{\prime}+L-K, N^{\prime}+L-K\right)$ entry being one. Applying (19)-(21) into (16)-(18) and with the assumption on the noise vector $\mathbf{w}_{i}^{(k)}$, it follows that

$$
\begin{aligned}
\mathbf{R}_{y}(0) & =E\left\{\mathbf{y}_{i}^{\left(L_{\mathrm{CP}}\right)} \mathbf{y}_{i}^{\left(L_{\mathrm{CP}}\right)^{*}}\right\} \\
& =\mathbf{H} \mathbf{H}^{*}+\sigma^{2} \mathbf{I}_{M N^{\prime}} \\
\mathbf{R}_{y}(K) & =E\left\{\mathbf{y}_{i}^{\left(L_{\mathrm{CP}}+K\right)} \mathbf{y}_{i}^{\left(L_{\mathrm{CP}}\right)^{*}}\right\} \\
& =\mathbf{H}\left(\mathbf{I}_{P} \otimes \mathbf{J}^{K}\right) \mathbf{H}^{*}+\sigma^{2}\left(\mathbf{I}_{M} \otimes \overline{\mathbf{J}}^{K}\right) \\
\mathbf{R}_{y}(-K) & =E\left\{\mathbf{y}_{i}^{\left(L_{\mathrm{CP}}\right)} \mathbf{y}_{i}^{\left(L_{\mathrm{CP}}+K\right)^{*}}\right\} \\
& =\mathbf{H}\left(\mathbf{I}_{P} \otimes \mathbf{J}^{-K}\right) \mathbf{H}^{*}+\sigma^{2}\left(\mathbf{I}_{M} \otimes \overline{\mathbf{J}}^{-K}\right)
\end{aligned}
$$

$$
\begin{aligned}
\mathbf{J}^{-K} \mathbf{J}^{K}= & {\left[\begin{array}{cc}
\mathbf{I}_{\left(N^{\prime}+L-K\right)} & \mathbf{0}_{\left(N^{\prime}+L-K\right) \times K} \\
\mathbf{0}_{K \times\left(N^{\prime}+L-K\right)} & \mathbf{0}_{K \times K}
\end{array}\right] } \\
\mathbf{J}^{-K} \mathbf{J}^{K}-\mathbf{J}^{-(K+1)} \mathbf{J}^{(K+1)}= & {\left[\begin{array}{ccc}
\mathbf{0}_{\left(N^{\prime}+L-K-1\right) \times\left(N^{\prime}+L-K-1\right)} & \mathbf{0}_{\left(N^{\prime}+L-K-1\right) \times 1} & \mathbf{0}_{\left(N^{\prime}+L-K-1\right) \times K} \\
\mathbf{0}_{1 \times\left(N^{\prime}+L-K-1\right)} & 1 & \mathbf{0}_{1 \times K} \\
\mathbf{0}_{K \times\left(N^{\prime}+L-K-1\right)} & \mathbf{0}_{K \times 1} & \mathbf{0}_{K \times K}
\end{array}\right] }
\end{aligned}
$$


where $\overline{\mathbf{J}}^{K}$ is an $N^{\prime} \times N^{\prime}$ matrix with the same structure as the matrix $\mathbf{J}^{K}$. From (24), it is observed that the noise variance $\sigma^{2}$ is the smallest eigenvalue of the matrix $\mathbf{R}_{y}(0)$ [7]. Therefore, it could be estimated from $\mathbf{R}_{y}(0)$, and the noise effect could be eliminated from the autocorrelation matrices (24)-(26). Consequently, the autocorrelation matrices without noise contribution are

$$
\begin{aligned}
\overline{\mathbf{R}}_{y}(0) & =\mathbf{R}_{y}(0)-\sigma^{2} \mathbf{I}_{M N^{\prime}}=\mathbf{H H}^{*} \\
\overline{\mathbf{R}}_{y}(K) & =\mathbf{R}_{y}(K)-\sigma^{2}\left(\mathbf{I}_{M} \otimes \overline{\mathbf{J}}^{K}\right) \\
& =\mathbf{H}\left(\mathbf{I}_{P} \otimes \mathbf{J}^{K}\right) \mathbf{H}^{*} \\
\overline{\mathbf{R}}_{y}(-K) & =\mathbf{R}_{y}(-K)-\sigma^{2}\left(\mathbf{I}_{M} \otimes \overline{\mathbf{J}}^{-K}\right) \\
& =\mathbf{H}\left(\mathbf{I}_{P} \otimes \mathbf{J}^{-K}\right) \mathbf{H}^{*} .
\end{aligned}
$$

Comparing the structures of the autocorrelation matrices in (27)-(29) with that of single-carrier MIMO systems in [28], it is easily seen that they are similar. It follows that the equalization method based on the autocorrelation matrices for singlecarrier MIMO systems in [28] can be readily applied here. Using (27)-(29), an equalizer is thus designed for ICI and ISI cancellation as

$$
\mathbf{G}_{K}=\mathbf{U}_{K}-\mathbf{U}_{K+1}
$$

where

$$
\mathbf{U}_{K}=\overline{\mathbf{R}}_{y}(-K) \overline{\mathbf{R}}_{y}(0)^{\#} \overline{\mathbf{R}}_{y}(K) \overline{\mathbf{R}}_{y}(0)^{\#} .
$$

Using Property 2 and (27)-(29), the matrix $\mathbf{U}_{K}$ is equivalent to

$$
\begin{aligned}
\mathbf{U}_{K} & =\overline{\mathbf{R}}_{y}(-K) \overline{\mathbf{R}}_{y}(0)^{\#} \overline{\mathbf{R}}_{y}(K) \overline{\mathbf{R}}_{y}(0)^{\#} \\
& =\mathbf{H}\left(\mathbf{I}_{P} \otimes \mathbf{J}^{-K}\right) \mathbf{H}^{*}\left(\mathbf{H H}^{*}\right)^{\#} \mathbf{H}\left(\mathbf{I}_{P} \otimes \mathbf{J}^{K}\right) \mathbf{H}^{*}\left(\mathbf{H} \mathbf{H}^{*}\right)^{\#} \\
& =\mathbf{H}\left(\mathbf{I}_{P} \otimes \mathbf{J}^{-K} \mathbf{J}^{K}\right) \mathbf{H}^{*}\left(\mathbf{H H}^{*}\right)^{\#}
\end{aligned}
$$

Substituting (23) and (32) into (30), the equalizer becomes equal to (33), shown at the bottom of the page, where $\mathbf{H}_{p}(a)$ denotes the $a$ th column of $\mathbf{H}_{p}$ in (9).
Applying the equalizer to the received signal vector $\mathbf{y}_{i}^{(k)}=\mathbf{H} \mathbf{x}_{i}^{(k)}+\mathbf{w}_{i}^{(k)}(7)$ and using Property $2,(10),(11)$, and (33), it is easy to obtain the equalizer output as

$$
\begin{aligned}
\mathbf{o}_{i}^{(k)} & =\mathbf{G}_{K} \mathbf{y}_{i}^{(k)} \\
& =\sum_{p=1}^{P} \mathbf{H}_{p}\left(N^{\prime}+L-K\right) s_{i, p}\left[N^{\prime}-1-k-K\right]+\boldsymbol{\eta}_{i}^{(k)} \\
& =\overline{\mathbf{H}}_{\mathrm{part}}^{(K)} \mathbf{s}_{i}\left[N^{\prime}-1-k-K\right]+\boldsymbol{\eta}_{i}^{(k)}, \quad k=0, \pm 1, \pm 2, \ldots
\end{aligned}
$$

where $\boldsymbol{\eta}_{i}^{(k)}=\mathbf{G}_{K} \mathbf{w}_{i}^{(k)}$, and we have (35) and (36), shown at the bottom of the page. Obviously, only one sampled transmitted signal $s_{i, p}\left[N^{\prime}-1-k-K\right]$ from each transmit antenna remains at the equalizer output. It turns out that both the ICI and ISI are completely eliminated. This is in contrast with the timedomain statistics-based technique [24], in which two sampled transmitted signals from each transmit antenna remain and only partial ICI and ISI cancellation is achieved in the first step of equalization.

From the derivation of the blind equalizer, it is apparent that there is no restriction between the channel length $L$ and the CP length $L_{\mathrm{CP}}$, which means that the blind equalization is applicable to general MIMO-OFDM systems, irrespective of whether the $\mathrm{CP}$ length is longer than, equal to, or shorter than the channel length.

\section{B. One-Tap Equalization}

Since the channel matrix $\mathbf{H}$ is assumed to be of full column rank after removing all-zero columns and the structure of $\mathbf{H}$ in (8) and (9) shows that the column $\mathbf{H}_{p}\left(N^{\prime}+L-K\right)$ is a nonzero column, the matrix $\overline{\mathbf{H}}_{\text {part }}^{(K)}$ in (36) is of full column rank. From (34), signal detection is easily achieved by

$$
\mathbf{s}_{i}\left[N^{\prime}-1-k-K\right] \approx \mathbf{G}_{\text {one-tap }} \mathbf{o}_{i}^{(k)}, \quad k=0, \pm 1, \pm 2, \ldots
$$

$$
\begin{aligned}
& \mathbf{G}_{K}=\mathbf{U}_{K}-\mathbf{U}_{K+1} \\
& =\mathbf{H}\left(\mathbf{I}_{P} \otimes\left(\mathbf{J}^{-K} \mathbf{J}^{K}-\mathbf{J}^{-(K+1)} \mathbf{J}^{(K+1)}\right)\right) \mathbf{H}^{*}\left(\mathbf{H H}^{*}\right)^{\#} \\
& =\left[\begin{array}{lllllll}
\mathbf{0}_{M N^{\prime} \times\left(N^{\prime}+L-K-1\right)} & \mathbf{H}_{1}\left(N^{\prime}+L-K\right) & \mathbf{0}_{M N^{\prime} \times K} & \cdots & \mathbf{0}_{M N^{\prime} \times\left(N^{\prime}+L-K-1\right)} & \mathbf{H}_{P}\left(N^{\prime}+L-K\right) & \mathbf{0}_{M N^{\prime} \times K}
\end{array}\right] \\
& \mathbf{H}^{*}\left(\mathbf{H H}^{*}\right)^{\#}
\end{aligned}
$$

$$
\begin{aligned}
\mathbf{s}_{i}[k] & =\left[\begin{array}{llll}
s_{i, 1}[k] & s_{i, 2}[k] & \cdots & s_{i, P}[k]
\end{array}\right]^{T} \\
\overline{\mathbf{H}}_{\text {part }}^{(K)} & =\left[\begin{array}{llll}
\mathbf{H}_{1}\left(N^{\prime}+L-K\right) & \mathbf{H}_{2}\left(N^{\prime}+L-K\right) & \cdots & \mathbf{H}_{P}\left(N^{\prime}+L-K\right)
\end{array}\right]
\end{aligned}
$$


where $\mathbf{G}_{\text {one-tap }}$ is a one-tap equalizer given by $\mathbf{G}_{\text {one-tap }}=$

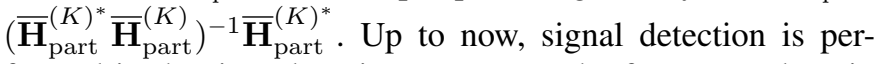
formed in the time domain. To recover the frequency-domain signals $\beta_{i, p}[n], n=0,1, \ldots, N-1, N$-point DFT must be performed to the time-domain signals $b_{i, p}[n], n=0,1, \ldots$, $N-1$, which is equal to the detected signals $\left(s_{i, p}\left[L_{\mathrm{CP}}\right], \ldots\right.$, $\left.s_{i, p}\left[N^{\prime}-1\right]\right)$ and is obtained from (37) by setting $k=N^{\prime}-$ $1-K-L_{\mathrm{CP}}, \ldots,-K$.

It is clear that the matrix $\overline{\mathbf{H}}_{\text {part }}^{(K)}$ needs to be estimated in the aforementioned signal detection technique. From (34), let

$$
\begin{aligned}
& \mathbf{O}_{\text {pilot }} \\
& \quad=\left[\begin{array}{llll}
\mathbf{o}_{i}^{\left(N^{\prime}-1-K-L_{\mathrm{CP}}\right)} & \mathbf{o}_{i}^{\left(N^{\prime}-1-K-L_{\mathrm{CP}}-1\right)} & \cdots & \mathbf{o}_{i}^{(-K)}
\end{array}\right]
\end{aligned}
$$

$\mathbf{S}_{\text {pilot }}$

$$
=\left[\begin{array}{llll}
\mathbf{s}_{i}\left[L_{\mathrm{CP}}\right] & \mathbf{s}_{i}\left[L_{\mathrm{CP}}+1\right] & \cdots & \mathbf{s}_{i}\left[N^{\prime}-1\right]
\end{array}\right]
$$

$\Gamma_{\text {pilot }}$

$$
=\left[\begin{array}{llll}
\boldsymbol{\eta}_{i}^{\left(N^{\prime}-1-K-L_{\mathrm{CP}}\right)} & \boldsymbol{\eta}_{i}^{\left(N^{\prime}-1-K-L_{\mathrm{CP}}-1\right)} & \cdots & \boldsymbol{\eta}_{i}^{(-K)}
\end{array}\right]
$$

where $\mathbf{S}_{\text {pilot }}$ is a pilot OFDM symbol. It follows that

$$
\mathbf{O}_{\text {pilot }}=\overline{\mathbf{H}}_{\text {part }}^{(K)} \mathbf{S}_{\text {pilot }}+\boldsymbol{\Gamma}_{\text {pilot }}
$$

and the matrix $\overline{\mathbf{H}}_{\text {part }}^{(K)}$ can be estimated using the least squares method as

$$
\overline{\mathbf{H}}_{\text {part }}^{(K)} \approx \mathbf{O}_{\text {pilot }} \mathbf{S}_{\text {pilot }}^{*}\left(\mathbf{S}_{\text {pilot }} \mathbf{S}_{\text {pilot }}^{*}\right)^{-1}
$$

In general, the number of subcarriers $N$ in one OFDM symbol is far greater than the number of transmit antennas $P$, and the pilot matrix $\mathbf{S}_{\text {pilot }}$ can be assumed to be of full row rank.

\section{Simulation Results}

Computer simulation is now conducted to investigate the performance of the proposed semi-blind time-domain equalization technique. In the following, a MIMO-OFDM system with $P=2$ transmit antennas and $M=3$ receive antennas is considered as an example. The transmitted signals are quadrature phase-shift keying (QPSK) modulated. The number of subcarriers in each OFDM symbol is $N=32$, and the length of CP is $L_{\mathrm{CP}}=8$. One pilot OFDM symbol is inserted at the beginning of each data packet with 500 OFDM symbols. The frequencyselective fading channel responses are randomly generated with a Rayleigh probability distribution and are static over each data packet. The parameter $K$ is set as 8 , unless otherwise indicated.

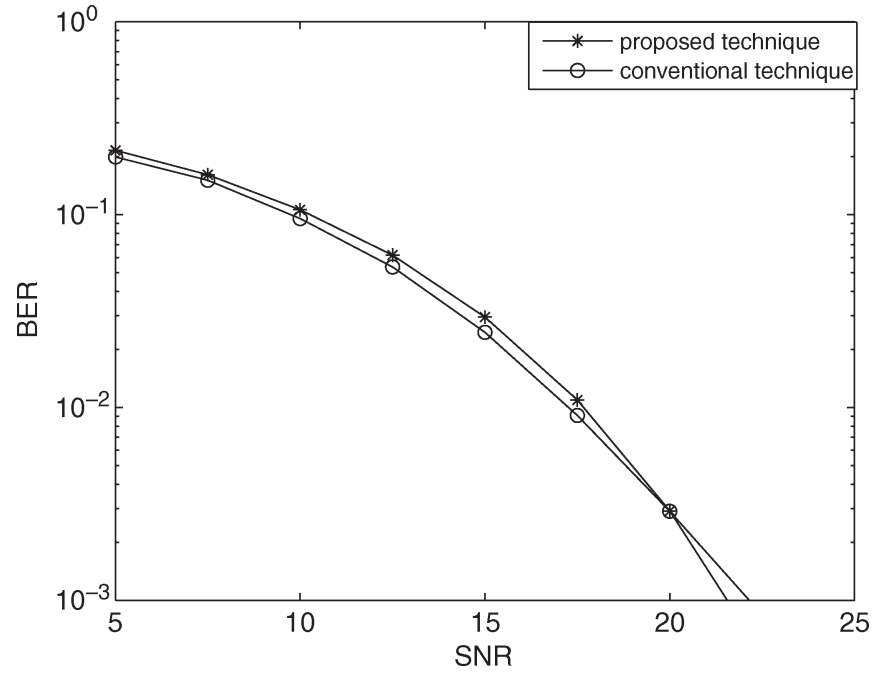

Fig. 3. BER versus SNR for $L=6\left(L \leq L_{\mathrm{CP}}\right)$.

The autocorrelation matrices $\mathbf{R}_{y}(0), \mathbf{R}_{y}(K)$, and $\mathbf{R}_{y}(-K)$ are computed as follows:

$$
\begin{aligned}
\mathbf{R}_{y}(0) & =E\left\{\mathbf{y}_{i}^{\left(L_{\mathrm{CP}}\right)} \mathbf{y}_{i}^{\left.\left(L_{\mathrm{CP}}\right)^{*}\right\}}\right. \\
& \approx \frac{1}{N_{s}} \sum_{i=1}^{N_{s}} \mathbf{y}_{i}^{\left(L_{\mathrm{CP}}\right)} \mathbf{y}_{i}^{\left(L_{\mathrm{CP}}\right)^{*}} \\
\mathbf{R}_{y}(K) & =E\left\{\mathbf{y}_{i}^{\left(L_{\mathrm{CP}}+K\right)} \mathbf{y}_{i}^{\left(L_{\mathrm{CP}}\right)^{*}}\right\} \\
& \approx \frac{1}{N_{s}} \sum_{i=1}^{N_{s}} \mathbf{y}_{i}^{\left(L_{\mathrm{CP}}+K\right)} \mathbf{y}_{i}^{\left(L_{\mathrm{CP}}\right)^{*}} \\
\mathbf{R}_{y}(-K) & =E\left\{\mathbf{y}_{i}^{\left(L_{\mathrm{CP}}\right)} \mathbf{y}_{i}^{\left(L_{\mathrm{CP}}+K\right)^{*}}\right\} \\
& \approx \frac{1}{N_{s}} \sum_{i=1}^{N_{s}} \mathbf{y}_{i}^{\left(L_{\mathrm{CP}}\right)} \mathbf{y}_{i}^{\left(L_{\mathrm{CP}}+K\right)^{*}}
\end{aligned}
$$

where $N_{s}$ is the number of OFDM symbols used and is set as 500, unless otherwise indicated. The signal-to-noise ratio (SNR) is defined as the ratio of the total received signal power with the total noise power, i.e.,

$$
\mathrm{SNR}=\frac{\sum_{m=1}^{M} E\left\{\left|\sum_{p=1}^{P} \sum_{l=0}^{L} h_{p m}(l) s_{i, p}[n-l]\right|^{2}\right\}}{\sum_{m=1}^{M} E\left\{\left|w_{i, m}[n]\right|^{2}\right\}} .
$$

The results shown in the following are averaged over 100 Monte Carlo realizations:

\section{A. Case With $L \leq L_{\mathrm{CP}}$}

In this case, the effect of frequency-selective fading can be completely eliminated by the standard OFDM technique, and the signals can be recovered by a one-tap frequency-domain equalizer on each subcarrier. Fig. 3 illustrates the comparison of the proposed technique with the conventional technique, 


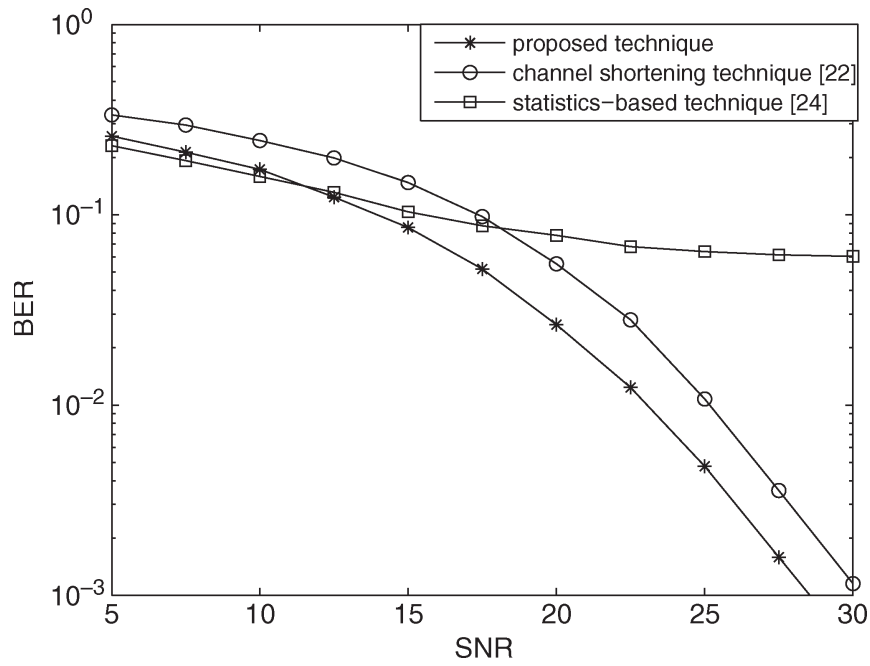

Fig. 4. BER versus SNR for $L=10\left(L>L_{\mathrm{CP}}\right)$.

which performs least squares equalization on each subcarrier after DFT. Results show that the performance of the proposed technique is close to that of the conventional technique and confirm that the blind equalizer designed in the first step can completely suppress both ICI and ISI, which are present in the time-domain signals.

\section{B. Case With $L>L_{\mathrm{CP}}$}

Since the channel length is longer than the CP length, the effect of frequency-selective fading cannot be completely eliminated by the standard OFDM technique, and ICI and ISI are introduced. For comparison, the time-frequency domain equalization technique with channel shortening [22] and the timedomain statistics-based technique [24] are also implemented. Results are shown in Fig. 4. Note that knowledge of the channel responses is assumed to be exactly known in the technique of Al-Dhahir [22] and two pilot OFDM symbols are utilized in the technique of $\mathrm{Ma}$ and $\mathrm{Ng}$ [24].

Obviously, the proposed technique outperforms the timefrequency domain technique with channel shortening [22]. For example, it achieves a bit-error-rate (BER) performance of $10^{-2}$ at $\mathrm{SNR}=23 \mathrm{~dB}$, whereas the time-frequency domain technique can only do so at $\mathrm{SNR}=25 \mathrm{~dB}$, indicating a $2-\mathrm{dB}$ improvement. The poorer performance of the technique in [22] is due to the residual ICI and ISI introduced by shortening the MIMO channels.

Compared with the time-domain statistics-based technique [24], the performance of the two are close for SNR $\leq 11 \mathrm{~dB}$, whereas the proposed technique is far superior to that of [24] for SNR $>11 \mathrm{~dB}$. The explanation is as follows: The timedomain signals are detected after ICI and ISI are eliminated by blind equalization in the proposed technique, whereas there are residual ICI and ISI in the technique of [24]. When the SNR is low, the additive noise dominates; hence, the performances of the two techniques are similar. The slightly better performance of the technique in [24] is probably due to two pilot OFDM symbols, instead of one, being used. When the SNR is high, the residual ICI and ISI in [24] limit its performance, whereas

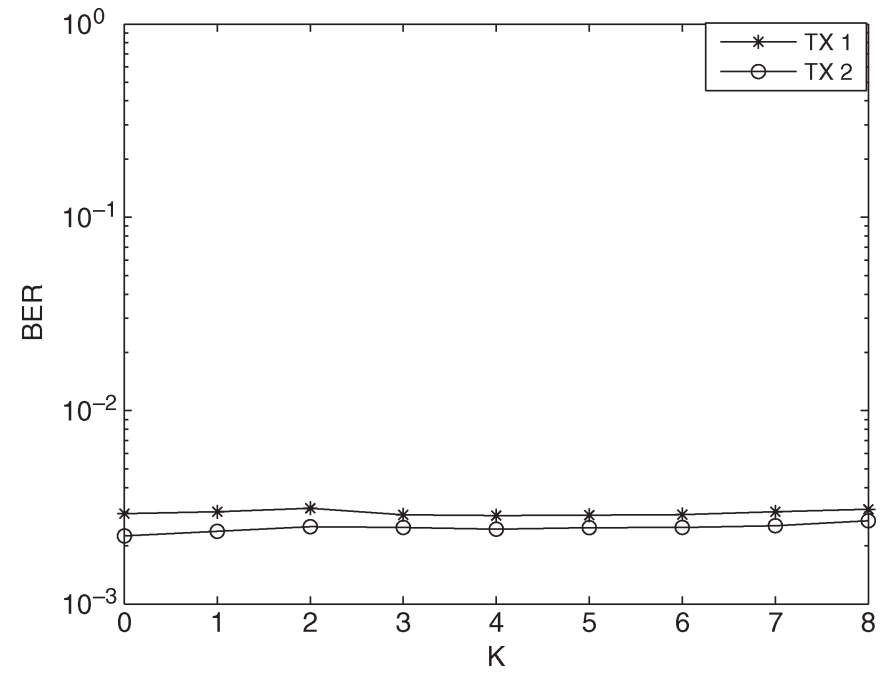

Fig. 5. Effect of $K$ for $L=6\left(L \leq L_{\mathrm{CP}} ; \mathrm{SNR}=20 \mathrm{~dB}\right)$.

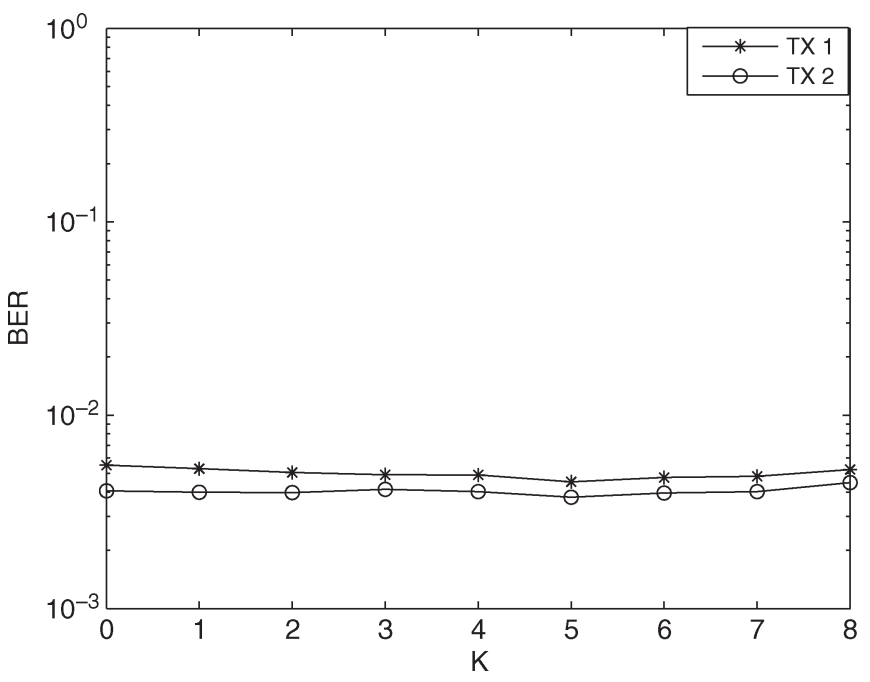

Fig. 6. Effect of $K$ for $L=10\left(L>L_{\mathrm{CP}} ; \mathrm{SNR}=25 \mathrm{~dB}\right)$.

the proposed technique follows a typical BER versus SNR behavior.

\section{Effect of the Parameter $K$}

In the proposed technique, only one parameter $K$, which represents the number of shifts in excess of the $\mathrm{CP}$ length, can be varied. It is set from 0 to 8 to test its effect on the proposed technique. Results are shown in Figs. 5 and 6 for $L \leq L_{\mathrm{CP}}$ and $L>L_{\mathrm{CP}}$ cases, respectively. It is obvious that the performance of the proposed technique is insensitive to the parameter $K$. This is an advantage over the time-frequency domain techniques, which are sensitive to the channel-shortening equalizer length and the delay.

\section{Effect of the Data Length $N_{s}$}

The blind equalizer in the proposed technique is designed using the autocorrelation matrices $\left[\mathbf{R}_{y}(0), \mathbf{R}_{y}(K)\right.$, and $\mathbf{R}_{y}(-K)$ ]. In practice, they are computed from a finite number of OFDM symbols, and the number of symbols used, i.e., $N_{s}$, 


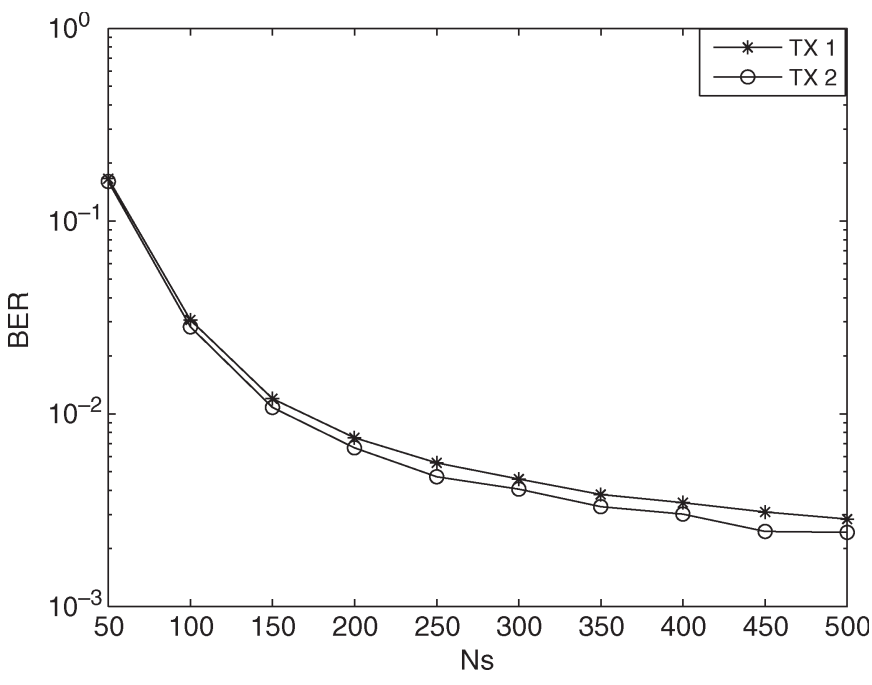

Fig. 7. Effect of $N_{s}$ for $L=6\left(L \leq L_{\mathrm{CP}} ; \mathrm{SNR}=20 \mathrm{~dB}\right)$.

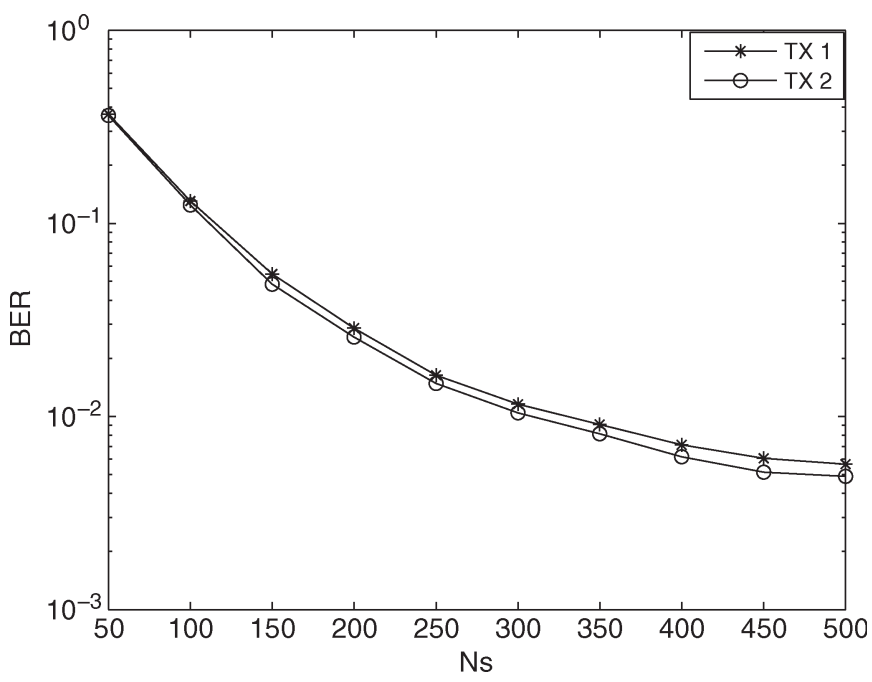

Fig. 8. Effect of $N_{s}$ for $L=10\left(L>L_{\mathrm{CP}} ; \mathrm{SNR}=25 \mathrm{~dB}\right)$.

may affect the performance. Figs. 7 and 8 show its performance when $N_{s}$ varies from 50 to 500 for $L \leq L_{\mathrm{CP}}$ and $L>L_{\mathrm{CP}}$ cases, respectively. As expected, better performance is achieved when more symbols are used.

\section{CONCLUSION}

In this paper, a semi-blind time-domain equalization technique has been proposed for general MIMO-OFDM systems, irrespective of whether the $\mathrm{CP}$ length is longer than/equal to or shorter than the channel length. A blind equalizer has been designed using the SOS of the received OFDM symbols shifted by more than or equal to the CP length to completely suppress both ICI and ISI. The time-domain signals are then detected from the blind equalizer output with the aid of only one pilot OFDM symbol. Simulations have demonstrated that the proposed technique is effective in suppressing ICI and ISI and robust against the number of shifts in excess of the CP length.

\section{REFERENCES}

[1] A. J. Paulraj, D. A. Gore, R. U. Nabar, and H. Bolcskei, "An overview of MIMO communications-A key to gigabit wireless," Proc. IEEE, vol. 92, no. 2, pp. 198-218, Feb. 2004.

[2] J. H. Winters, J. Salz, and R. D. Gitlin, "The impact of antenna diversity on the capacity of wireless communication systems," IEEE Trans. Commun., vol. 42, no. 2-4, pp. 1740-1751, Feb.-Apr. 1994.

[3] D. Gesbert, M. Shafi, D. Shiu, P. J. Smith, and A. Naguib, "From theory to practice: An overview of MIMO space-time coded wireless systems," IEEE J. Sel. Areas Commun., vol. 21, no. 3, pp. 281-302, Apr. 2003.

[4] H. Dai, A. F. Molisch, and H. V. Poor, "Downlink capacity of interferencelimited MIMO systems with joint detection," IEEE Trans. Wireless Commun., vol. 3, no. 2, pp. 442-453, Mar. 2004.

[5] B. Muquet, Z. Wang, G. B. Giannakis, M. de Courville, and P. Duhamel, "Cyclic prefixing or zero padding for wireless multicarrier transmissions?" IEEE Trans. Commun., vol. 50, no. 12, pp. 2136-2148, Dec. 2002.

[6] J. Lei and T. S. Ng, "A consistent OFDM carrier frequency offset estimator based on distinctively spaced pilot tones," IEEE Trans. Wireless Commun., vol. 3, no. 2, pp. 588-599, Mar. 2004.

[7] Y. Zeng and T. S. Ng, "A semi-blind channel estimation method for multiuser multiantenna OFDM systems," IEEE Trans. Signal Process., vol. 52, no. 5, pp. 1419-1429, May 2004.

[8] C. Dubuc, D. Starks, T. Creasy, and Y. Hou, "A MIMO-OFDM prototype for next-generation wireless WANs," IEEE Commun. Mag., vol. 42, no. 12, pp. 82-87, Dec. 2004.

[9] A. Van Zelst and T. C. W. Schenk, "Implementation of a MIMO OFDMbased wireless LAN system," IEEE Trans. Signal Process., vol. 52, no. 2, pp. 483-494, Feb. 2004.

[10] K. J. Kim, J. Yue, R. A. Iltis, and J. D. Gibson, "A QRD-M/Kalman filter-based detection and channel estimation algorithm for MIMO-OFDM systems," IEEE Trans. Wireless Commun., vol. 4, no. 2, pp. 710-721, Mar. 2005.

[11] L. Giangaspero, L. Agarossi, G. Paltenghi, S. Okamura, M. Okada, and S. Komaki, "Co-channel interference cancellation based on MIMO OFDM systems," IEEE Wireless Commun., vol. 9, no. 6, pp. 8-17, Dec. 2002.

[12] S. Thoen, L. Deneire, L. Van der Perre, M. Engels, and H. De Man, "Constrained least squares detector for OFDM/SDMA-based wireless networks," IEEE Trans. Wireless Commun., vol. 2, no. 1, pp. 129-140, Jan. 2003

[13] W. Yan, S. Sun, and Z. Lei, "A low complexity VBLAST OFDM detection algorithm for wireless LAN systems," IEEE Commun. Lett., vol. 8, no. 6, pp. 374-376, Jun. 2004.

[14] S. Y. Park and C. G. Kang, "Complexity-reduced iterative MAP receiver for interference suppression in OFDM-based spatial multiplexing systems," IEEE Trans. Veh. Technol., vol. 53, no. 5, pp. 1316-1326, Sep. 2004.

[15] K. B. Letaief, E. Choi, J. Y. Ahn, and R. Chen, "Joint maximum likelihood detection and interference cancellation for MIMO/OFDM systems," in Proc. IEEE VTC-Fall, 2003, pp. 612-616.

[16] J. Wang and B. Daneshrad, "Performance of linear interpolation-based MIMO detection for MIMO-OFDM systems," in Proc. IEEE WCNC, 2004, pp. 981-986.

[17] Y. Li, J. H. Winters, and N. R. Sollenberger, "MIMO-OFDM for wireless communications: Signal detection with enhanced channel estimation," IEEE Trans. Commun., vol. 50, no. 9, pp. 1471-1477, Sep. 2002.

[18] K. Van Acker, G. Leus, M. Moonen, O. van de Wiel, and T. Pollet, "Per tone equalization for DMT-based systems," IEEE Trans. Commun., vol. 49, no. 1, pp. 109-119, Jan. 2001

[19] G. Leus and M. Moonen, "Per-tone equalization for MIMO OFDM systems," IEEE Trans. Signal Process., vol. 51, no. 11, pp. 2965-2975, Nov. 2003.

[20] P. J. W. Melsa, R. C. Younce, and C. E. Rohrs, "Impulse response shortening for discrete multitone transceivers," IEEE Trans. Commun., vol. 44, no. 12, pp. 1662-1672, Dec. 1996.

[21] T. Tang and R. W. Heath, Jr., "Space-time interference cancellation in MIMO-OFDM systems," IEEE Trans. Veh. Technol., vol. 54, no. 5, pp. 1802-1816, Sep. 2005.

[22] N. Al-Dhahir, "FIR channel-shortening equalizers for MIMO ISI channels," IEEE Trans. Commun., vol. 49, no. 2, pp. 213-218, Feb. 2001.

[23] D. Daly, C. Heneghan, and A. D. Fagan, "A minimum mean-squared error interpretation of residual ISI channel shortening for discrete multitone transceivers," in Proc. IEEE ICASSP, 2001, pp. 2065-2068. 
[24] S. D. Ma and T. S. Ng, "Time domain signal detection based on secondorder statistics for MIMO-OFDM systems," IEEE Trans. Signal Process., vol. 55, no. 3, pp. 1150-1158, Mar. 2007.

[25] J. Zhang and W. Ser, "Delay selection for TEQ in OFDM systems," in Proc. IEEE ISSPIT, 2003, pp. 443-446.

[26] J. Shen and Z. Ding, "Direct blind MMSE channel equalization based on second-order statistics," IEEE Trans. Signal Process., vol. 48, no. 4, pp. 1015-1022, Apr. 2000.

[27] A. Gorokhov and P. Loubaton, "Subspace-based techniques for blind separation of convolutive mixtures with temporally correlated sources," IEEE Trans. Circuits Syst. I, Fundam. Theory Appl., vol. 44, no. 9, pp. 813-820, Sep. 1997.

[28] J. Zhu, Z. Ding, and X.-R. Cao, "Column-anchored zeroforcing blind equalization for multiuser wireless FIR channels," IEEE J. Sel. Areas Commun., vol. 17, no. 3, pp. 411-423, Mar. 1999.

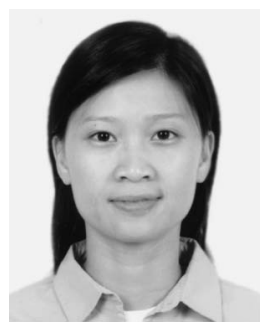

Shaodan Ma received the B.Sc.(Eng.) and M.Eng.Sc. degrees in electrical engineering from Nankai University, Tianjin, China, in 1999 and 2002, respectively, and the Ph.D. degree in electrical and electronic engineering from The University of Hong Kong, Hong Kong, in 2006.

Since 2006, she has been with the Department of Electrical and Electronic Engineering, The University of Hong Kong, as a Postdoctoral Fellow. Her research interests include wireless communication systems, spread-spectrum techniques, MIMO systems, OFDM techniques, and related digital signal processing.

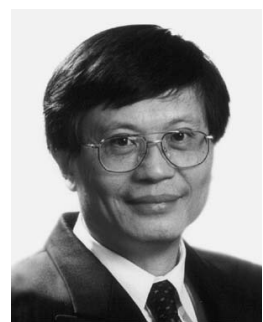

Tung-Sang Ng (F'03) received the B.Sc.(Eng.) degree in electrical engineering from The University of Hong Kong, Hong Kong, in 1972 and the M.Eng.Sc. and $\mathrm{Ph} . \mathrm{D}$. degrees in electrical engineering and the Honorary Doctor of Engineering degree from The University of Newcastle, Newcastle, Australia, in 1974, 1977, and 1997, respectively.

After graduation, he worked for BHP Steel International and the University of Wollongong, Wollongong, Australia, for 14 years before he returned to The University of Hong Kong in 1991, taking up the position of a Professor and the Chair of Electronic Engineering. He was the Head of the Department of Electrical and Electronic Engineering from 2000 to 2003 and the Dean of Engineering from 2003 to 2007. He has authored or coauthored more than 250 papers published in international journals and conference proceedings. His current research interests include wireless communication systems, spread-spectrum techniques, CDMA, and digital signal processing.

Prof. $\mathrm{Ng}$ is a Fellow of the Institution of Electrical Engineers (IEE) and the Hong Kong Institution of Engineers. He was the General Chair of the 1997 IEEE International Symposium on Circuits and Systems and the Vice President for Region 10 of the IEEE Circuits and Systems Society in 1999 and 2000. He was an Executive Committee Member and a Board Member of the IEE Informatics Divisional Board (1999-2001) and was an ordinary member of the IEE Council (1999-2001). He was the recipient of the Senior Croucher Foundation Fellowship in 1999, the IEEE Third Millenium Medal in 2000, and the Outstanding Researcher Award in 2003 from The University of Hong Kong. 